\title{
Hiperkalemia grave asociada a drogas que actúan sobre el sistema renina, angiotensina, aldosterona: Un problema que requiere atención. Caso clínico
}

\author{
Ronald Kauffmann Q, Rodrigo O rozco B, \\ Juan Carlos Venegas $\mathbf{G}$. \\ Severe hyperkalemia associated \\ to the use of losartan and \\ spironolactone. Case report
}

\begin{abstract}
Hyperkalemia is a complications of the use of angiotensin converting enzyme inhibitors, angiotensin receptor antagonists and aldosterone antagonists. These drugs are commonly used for the treatment of hypertension and cardiac failure. We report a 84 year-old female treated with losartan $50 \mathrm{mg} /$ day and spironolactone $25 \mathrm{mg} / \mathrm{day}$ that presented with a hyperkalemia of $8.4 \mathrm{mEq} / \mathrm{l}$ and bradicardia, drowsiness and respiratory depression. She required hemodialysis and ventilatory assistance. She was discharged in good conditions five days after admission (Rev Méd Chile 2005; 133: 947-52).
\end{abstract}

(Key Words: Hyperkalemia; Losartan; Spironolactone)

Recibido el 4 de marzo, 2005. Aceptado el 13 de abril, 2005.

Unidad de Cuidados Coronarios e Intermedio Coronario, Clínica Las Condes. Santiago de Chile.

$\mathrm{E}^{1}$ sistema renina angiotensina aldosterona (RAA) tiene un rol fisiológico trascendental en la regulación de la presión arterial a través de la homeostasis del agua, sodio y potasio. En pacientes con hipertensión arterial o con insuficiencia cardíaca, la activación patológica de este sistema contribuye significativamente en la génesis de daño cardíaco y vascular. Por este motivo, en los últimos 25 años, ha existido un gran esfuerzo para

Correspondencia a: Dr Ronald Kauffmann. Unidad de Cuidados Coronarios e Intermedio Coronario, Clínica Las Condes. Santiago de Chile. E mail: roka@clinicalascondes.cl intervenir farmacológicamente este eje en varios niveles ${ }^{1}$.

Los agentes inhibidores de la enzima convertidora (IECA), los antagonistas de los receptores de angiotensina II tipo I (ARAII) y los antagonistas de la aldosterona, se usan comúnmente en la práctica médica general como monoterapia y también asociados entre sí, para el tratamiento de un número creciente de pacientes con hipertensión arterial $^{2-4}$, insuficiencia cardíaca ${ }^{5-8}$, postinfarto agudo del miocardio ${ }^{9,10}$ o pacientes en general de alto riesgo cardiovascular ${ }^{11}$, con el objetivo de disminuir la morbimortalidad cardiovascular ${ }^{3-11}$. Además, en los pacientes diabéticos se ha emplea- 
do para retardar la aparición de la insuficiencia renal y la reducción de la proteinuria ${ }^{12-14}$.

En general, estos medicamentos son considerados como seguros y su utilidad en enfermedades de alta prevalencia explica por qué su uso es tan difundido. Sin embargo, el riesgo de hiperkalemia con estos agentes puede poner en peligro la vida del paciente ${ }^{15}$. Creemos que la frecuencia y severidad de esta complicación se ha subestimado, lo que nos motiva a comunicar el presente caso.

\section{CASO CLÍNICO}

Historia actual: mujer, 84 años, se mantenía en buenas condiciones generales, autovalente para sus actividades habituales. Ingresó el 31/12/04 a través de Servicio de Urgencia por presentar una semana antes un cuadro caracterizado por decaimiento y malestar general inespecífico. El día anterior a su ingreso presentó 3 episodios de deposiciones semilíquidas y dolor abdominal tipo cólico de intensidad leve. El día de su ingreso fue observada por su hijo médico en su domicilio quien la encontró comprometida de conciencia, con falta de reactividad a órdenes verbales, mirada fija, midriasis e hipoventilación que evoluciona a la apnea, por lo que fue sometida a reanimación básica, recuperando nivel de conciencia y ventilación espontánea.

Antecedentes mórbidos: angina de pecho estable de varios años de evolución, accidente cerebrovascular cerebeloso sin secuelas, 5 años antes. Linfoma tratado hace 5 años, sin recidiva desde hace 2 años. Hipertensión arterial (HTA) crónica de difícil manejo e insuficiencia mitral severa, con historia de disnea de medianos esfuerzos. Terapia habitual: carvedilol 6,25 mg/día, losartan $50 \mathrm{mg} /$ día, espironolactona $25 \mathrm{mg} /$ día, hidroclorotiazida $12,5 \mathrm{mg} /$ día, amlodipino $5 \mathrm{mg} /$ día. Mantenía controles médicos y de laboratorio en forma periódica. De sus exámenes previos, destacaba una creatinina basal de 1,2 mg/dl, clearence de creatinina 35-40 $\mathrm{ml} / \mathrm{min}$ y electrocardiograma en ritmo sinusal.

Examen físico: soporosa, sin déficit neurológico focal, presión arterial: 146/76 mmHg, pulso $30 \mathrm{a}$ 40 por $\min , \mathrm{Sa} 02>95 \%$. Pulso venoso yugular normal. Examen cardíaco: ruidos regulares, soplo holosistólico intensidad III/VI en foco mitral. Sin signos congestivos pulmonares, sin edema de extremidades. Abdomen depresible e indoloro.

Laboratorio al ingreso: electrocardiograma: ritmo idioventricular $50 / \mathrm{min}$, trastomo de la conducción intraventricular mal definido con QRS 0,12 s y ondas $\mathrm{T}$ aumentadas de voltaje. Electrólitos plasmáticos: K: 8,4 mEq/l, Cl: $109 \mathrm{mEq} / \mathrm{l}$, Na: $132 \mathrm{mEq} /, \mathrm{pH}$ 7,24, PCO2: 27, HCO3 de $12 \mathrm{mEq} /$, anion gap 10. BE: 14,4. Nitrógeno ureico $61 \mathrm{mg} / \mathrm{dl}$, creatinina 2,63 mg/ dl, hematocrito 33\%, hemoglobina $0,7 \mathrm{mg} / \mathrm{dl}$, glóbulos blancos 8.660, glicemia $137 \mathrm{mg} / \mathrm{dl}$, troponinas T $\varangle 0,01$, orina completa normal. Radiografía de tórax sin congestión pulmonar. Ecocardiograma: mixomatosis mitral y tricuspídea, con prolapso mitral e insuficiencia mitral y tricuspídea severas, hipertrofia ventricular izquierda concéntrica leve con función sistólica normal, dilatación biauricular moderada.

Evolución: no presentó diarrea durante la hospitalización. No hubo respuesta a las medidas médicas habituales para controlar la hiperkalemia, por lo que se efectuó hemodiálisis de emergencia después de su ingreso. Durante el procedimiento recuperó ritmo sinusal 102 por min, inicialmente hipertensa sistólica hasta 208/63 mmHg y luego con uso de propanolol mantuvo presiones normales, en estas circunstancias presentó angina de pecho con infradesnivel del segmento ST en el ECG y elevación de marcadores de daño miocárdico: CPK fracción MB hasta $8,3 \mathrm{mg} / \mathrm{dl}$, TT hasta 0,072 y mioglobina hasta $384 \mathrm{mg} / \mathrm{dl}$, el $\mathrm{K}$ había descendido a 4,7 mEq/L. Fracción excretada de Na 13\%, fracción excretada de nitrógeno ureico: $16 \%$. La paciente normalizó antes de las $24 \mathrm{~h}$ su potasio y diuresis, mantuvo cifras tensionales normales, con niveles de creatinina de $1,3 \mathrm{~m} / \mathrm{dl}$, nitrógeno ureico $24 \mathrm{mg} / \mathrm{dl}$ y fue dada de alta al quinto día en buenas condiciones.

\section{DisCUSIÓN}

Esta paciente de 84 años con hipertensión arterial crónica, cardiopatía hipertensiva, coronaria y valvular, estaba en tratamiento con 5 fármacos que tienen propiedades antihipertensivas, dos de ellos además antianginosos y 2 con efectos en el sistema 
RAA: losartan $50 \mathrm{mg} /$ día, que bloquea los receptores de angiotensina II y espironolactona $25 \mathrm{mg} /$ día, un antagonista competitivo de la aldosterona. Estos medicamentos estaban prescritos en las dosis habituales, en una paciente adherente. Presentó una hiperkalemia de $8,4 \mathrm{mEq} / \mathrm{l}$, que determinó bradicardia, compromiso de conciencia, depresión respiratoria con necesidad de reanimación ventilatoria inicial y posteriormente hemodiálisis de urgencia para la corrección definitiva de su cuadro. Además, presentó durante la diálisis un infarto agudo del miocardio sin supradesnivel del segmento ST, con moderada alza de los marcadores miocárdicos. Todas estas complicaciones pusieron en riesgo la vida de una paciente que se mantenía previamente en buenas condiciones generales. En este caso, creemos que la asociación de losartan y espironolactona tuvo un importante rol en el desarrollo de la hiperkalemia. Contribuyó también el déficit de volumen extracelular (VEC), aunque en este caso fue leve y la insuficiencia renal aguda premenal secundaria a diarrea transitoria. En los controles médicos previos, se comprobó una función renal estable con creatinina de $1,2 \mathrm{mg} / \mathrm{dl}$. La paciente también recibía hidroclorotiazida $12,5 \mathrm{mg}$, que es una dosis baja pero, en uso crónico podría haber facilitado el déficit de VEC previo a su cuadro de diarrea. Por otra parte, este diurético tiene el efecto de aumentar las pérdidas renales de sodio $\mathrm{y}$ potasio, por lo que es una asociación conveniente con IECA, ARA II o espironolactona, para evitar la hiperkalemia. Es de interés señalar que la constatación de hiperkalemia en una paciente con diarrea no es habitual, por la pérdida de potasio a través de las deposiciones ${ }^{16}$. En una publicación se hace hincapié en lo infrecuente de esta asociación, y se describen 4 casos de pacientes con diarrea e hiperkalemia que tenían en común estar en tratamiento con IECA ${ }^{17}$. Las causas más frecuentes de hiperkalemia se muestran en la Tabla 1.

A pesar que muchos de los efectos adversos de los medicamentos son conocidos, predecibles y evitables, éstos siguen ocurriendo con gran frecuencia. La interacción entre medicamentos puede explicar muchos de estos casos. Es necesario estar cada vez más alerta a las complicaciones que se producen por la asociación de medicamentos porque éste es un problema que va en aumento. Actualmente existen recomendaciones que estimulan la asociación de fármacos para el

\title{
Tabla 1. C ausas frecuentes de hiperkalemia
}

\author{
1. Aumento de la liberación de $\mathrm{K}$ desde las células \\ * Pseudohiperkalemia (trauma mecánico durante la punción venosa) \\ * Acidosis metabólica \\ * Déficit de insulina \\ * Aumento catabolismo celular \\ * Ejercicio \\ * Bloqueadores beta adrenérgicos \\ * Otros (sobredosis de digital, parálisis periódica hiperkalemica, succinilcolina) \\ 2. Aumento del aporte \\ * En soluciones parenterales \\ * Ingesta oral en jugos y suplementos orales de potasio \\ 3. Disminución de la excreción de potasio \\ * Insuficiencia renal \\ * Disminución del volumen circulante efectivo \\ * Hipoaldosteronismo hiporeninémico (ATR-IV) \\ * Drogas: \\ Inhibidores de la enzima convertidora de angiotensina \\ Bloqueadores de los receptores de angiotensina II \\ Espironolactona, eplerenona
}


tratamiento de una misma condición, por ejemplo, hipertensión arterial e insuficiencia cardíaca, debido a que se consiguen efectos benéficos aditivos. Por otra parte, el envejecimiento de la población trae como consecuencia la comorbilidad que requiere medicamentos para el tratamiento de diversas enfermedades crónicas y además es cada vez más difundido el uso de drogas con el objetivo de prevención primaria.

Los medicamentos que bloquean el sistema RAA benefician a pacientes con hipertensión arterial, disfunción ventricular izquierda sintomática 0 asintomática, postinfarto agudo del miocardio, diabéticos y nefrópatas. Los efectos más destacados son la reducción de la presión arterial, masa ventricular izquierda, proteinuria y de la morbimortalidad cardiovascular2-14.

Agregar espironolactona a un paciente que está recibiendo en forma prolongada un IECA tiene su justificación, los IECA no suprimen la producción de aldosterona en forma completa y permanente, esto se ha denominado escape de aldosterona ${ }^{18,19}$. En el estudio Randomized Aldactone Evaluation Study (RALES), se confirmó una reducción adicional en la morbimortalidad cardiovascular al agregar espironolactona al tratamiento habitual en pacientes con insuficiencia cardíaca avanzada ${ }^{8}$.

El riesgo de hiperkalemia en pacientes que reciben fármacos que inhiben el eje RAA es un hecho que está suficientemente descrito, tanto en monoterapia y con mayor razón con su asociación, aun en dosis terapéuticas. Es probable que en la práctica médica habitual exista una subestimación de esta complicación. Los ensayos randomizados comunican una baja incidencia de hiperkalemia, por ejemplo, en el estudio RALES 8 , con espironolactona, alcanzó solamente a $2 \%$ y en el estudio The Eplerenone PostAMI Heart Failure Efficacy and Survival Study (EPHESUS) con eplerenona, que es otro bloqueador selectivo de la aldosterona, fue $5,5 \%{ }^{20}$. En el estudio The Valsartan in Acute Myocardial Infarction Trial (VALANT) la incidencia de hiperkalemia en pacientes tratados con valsartan, captopril y valsartan más captopril fue de $1,4 \%, 1 \%$ y $1,4 \%$, respectivamente ${ }^{21}$. Esto ha contribuido a la percepción de seguridad de estos fármacos. Sin embargo, la baja incidencia de hiperkalemia en los ensayos controlados puede ser atribuida en parte a la selección de pacientes de bajo riesgo, controles frecuentes en el seguimiento $\mathrm{y}$ monitorización protocolizada de los niveles de potasio y creatinina. Por ejemplo, en el primer ensayo no se reclutaron diabéticos insulino requirentes ni pacientes con creatinina $>2 \mathrm{mg} / \mathrm{dl}$, más llamativo es que la concentración promedio de los principales estudios de tratamiento de pacientes con insuficiencia cardíaca tenían un rango de creatininemia entre 1,2 y 1,4 mg/ $\mathrm{dl}^{22}$. Es de interés señalar que después de la publicación del RALES se comprobó en Ontario, Canadá, un abrupto incremento en la prescripción de espironolactona, que se asoció a un mayor número de hospitalización de pacientes con hiperkalemia y una mayor morbimortalidad por esta causa ${ }^{23}$.

En pacientes ambulatorios, la incidencia de hiperkalemia, definida como $\mathrm{K}>5,1 \mathrm{mEq} / \mathrm{l}$, durante el primer año de la indicación de drogas que actúan en el eje RAA, es aproximadamente $11 \% 24$. En los pacientes hospitalizados esta complicación es más frecuente y puede ocurrir hasta en $38 \% 25$. Los pacientes con mayor riesgo de presentar esta complicación son los sujetos mayores de 70 años, los diabéticos y en aquellos que existe de base un compromiso de la función renal con una filtración glomerular estimada $<30 \mathrm{ml}$ por min, en los diabéticos este umbral puede ser más alto. En pacientes sin factores de riesgo la hiperkalemia es infrecuente $22,24,25$.

La coexistencia de insuficiencia renal en pacientes con insuficiencia cardíaca es relativamente frecuente, hasta un tercio de los pacientes o hasta la mitad de aquellos con insuficiencia cardíaca severa tienen insuficiencia renal y, por lo tanto, riesgo de hiperkalemia. El dilema es que los pacientes con grados más avanzados de disfunción ventricular izquierda son justamente los que más se benefician con medicamentos que actúan en el eje RAA y también los más expuestos a los efectos adversos ${ }^{22,26}$.

Los riesgos de interacción de drogas aumentan excesivamente con el número de medicamentos utilizados, los pacientes que reciben 2 medicamentos tienen un riesgo de interacción de aproximadamente $15 \%$, para 5 medicamentos $40 \%$ y con 7 medicamentos $80 \%$. El adulto mayor está expuesto a mayor riesgo, representan el grupo etario de mayor consumo de medicamentos con un promedio de 2 a 5 al día ${ }^{27}$. De las combinaciones de medicamentos que altera la excreción de potasio destacan: antiinflamatorios no esteroidales, heparina, ketoconazol, trimetropin, ciclosporina, tacrolimus, entre otros ${ }^{22}$ (Tabla 2). 
Tabla 2. Factores de riesgo de hiperkalemia en pacientes que reciben drogas que actúan sobre $\mathrm{el}$ sistema renina, angiotensina, aldosterona*

1. Insuficiencia renal

2. Diabetes Mellitus

3. Insuficiencia cardíaca descompensada

4. Edad avanzada

5. Depleción de volumen extracelular

6. Asociación con otras drogas que alteran la excreción de potasio:

* Diuréticos retenedores de $\mathrm{K}$ : triamterene, amiloride

* Antiinflamatorios no esteroidales

* Inhibidores de calcineurina: ciclosporina, tacrolimus

* Heparina

* Ketoconazol

* Trimetropin

*Adaptado de referencia 22.

\section{REFERENCIAS}

1. Kamel KS, Quaggin S, Scheich A, Halperin ML. Disorders of potassium homeostasis: an approach based on pathophysiology. Am J Kidney Dis 1994; 24: 597-613.

2. Dahlöf B, Devereux RB, KJeldsen SE, Julus $S$, BeEvers G, DE Faire U et al. Cardiovascular morbidity and mortality in the Losartan Intervention for Endpoint reduction in hypertension study (LFE): a randomized trial against atenolol. Lancet 2002; 359: 995-1003.

3. Blood Pressure Lowering Treatment Trialists' ColaBORATION. Effects of ACE inhibitors, calcium antagonists, and other blood pressure-lowering drugs on mortality and major cardiovascular morbidity. Lancet 2000; 356: 1955-64.

4. BLACK HR. Evolving role of aldosterone blockers alone and in combination with angiotensinconverting enzyme inhibitors or angiotensin II receptor blockers in hypertension management: A review of mechanistic and clinical data. Am Heart J 2004; 147: 564-72.

5. The SOLVD InVESTIGATORS. Effect of enalapril on mortality and the development of heart failure in
Para evitar la hiperkalemia en pacientes que reciben drogas con efecto en el eje RAA, particularmente en aquellos de alto riesgo, se ha sugerido iniciar los medicamentos con la dosis recomendada más baja e incremento gradual, medir el $\mathrm{K}$ una vez a la semana después de iniciada la terapia y después de cada aumento de la dosis o al momento de su asociación con otro fármaco que retiene $\mathrm{K}$. Cuando la espironolactona se agrega a otros fármacos que actúan en sistema RAA, no se debe sobrepasar la dosis de $25 \mathrm{mg}$. Evitar la depleción de VEC y corregir precozmente cuando ésta se produce. Reconocer que un exceso de diuréticos es una causa frecuente de depleción de VEC, insuficiencia renal e hiperkalemia. Controlar periódicamente la creatinina y el $\mathrm{K}$ cuando se aumentan los diuréticos o existen pérdidas por vómitos, diarrea etc. $\mathrm{Si}$ existe un aumento de $\mathrm{K}$ a niveles $>5,5 \mathrm{mEq} / \mathrm{l}$ es necesario suspender los medicamentos 22,26 .

asymptomatic patients with reduced left ventricular ejection fractions. NEngl J Med 1992; 327: 68591.

6. Cohn JN, Tognoni G, the Valsartan Heart Failure TRIAL InVESTIGaTORS. A randomized trial of the angiotensin-receptor blocker valsartan in chronic heart failure. N Engl J Med 2001; 345: 1667-75.

7. Pfeffer ma, Swedberg $K$, Granger CB, Held $P$, McMurRay JJ, Michelson E et al. Effects of candesartan on mortality and morbidity in patients with chronic heart failure: the CHARM-Overall programme. Lancet 2003; 362: 759-66.

8. Pitt B, Zannad F, Remme WJ, Cody R, Castaigne A, PÉREZ A ET AL. The effect of spironolactone on morbidity and mortality in patients with severe heart failure. N Engl J Med 1999; 341: 709-17.

9. ACE InHibitor Myocardial Infarction CoLaborative GRoup. Indications for ACE inhibitors in the early treatment of acute myocardial infarction: systematic overview of individual data from 100,000 patients in randomized trials. Circulation 1998; 97: 2202-12.

10. DicKSTein K, KJeKSHUS J. Effects of losartan and captopril on mortality and morbidity in high-risk patients after acute myocardial infarction: the 
OPTIMAAL randomized trial: Optimal Trial in Myocardial Infarction with Angiotensin II Antagonist Losartan. Lancet 2002; 360: 752-60.

11. The Heart Outcomes Prevention Evaluation Study INVESTIGATORS. Effects of an angiotensin-converting enzyme inhibitor, ramipril, on cardiovascular events in high-risk patients: The Heart Outcomes Prevention Evaluation Study Investigators. N Engl J Med 2000; 342: 145-53.

12. Marre M, Chateluer G, Leblanc H et al. Prevention of diabetic nephropathy with enalapril in normotensive diabetics with microalbuminuria. BMJ 1988; 297: 1092-5.

13. Lewis EJ, Hunsicker LG, Clarke WR, Berl T, Pohl MA, LEWIS JB ET AL Renoprotective effect of the angiotensin-receptor antagonist irbesartan in patients with nephropathy due to type 2 diabetes. N Engl J Med 2001; 345: 851-60.

14. Brenner BM, Cooper ME, de Zeeuw D, Keane WF, Mitch WE, Parving H et al. Effects of losartan on renal and cardiovascular outcomes in patients with type 2 diabetes and nephropathy. N Engl J Med 2001; 345: 861-9.

15. Schepkens $H$, Vanholder R, Biшouw J, Lameire N. Life-threatening hyperkelemia during combined therapy with angiotensin-converting enzyme inhibitors and spironolactone: an analysis of 25 cases. Am J Med 2001; 110: 438-41.

16. YaP AS, Hockings GI, Boyle PD. Hyperkalemia associated with diarrhea. Ann Intern Med 1990; 112: 470.

17. Taylor JG, Zwiшch CW, Kaehny WD, Levi M, Popovtzer M. Hyperkalemia With Concomitant Watery Diarrhea: An Unusual Association. Am J Kidney Dis 2003; 42: E5.

18. Sato A, Saruta T. Aldosterone escape during angiotensin-converting enzyme inhibitor therapy in essential hypertensive patients with left ventricular hypertrophy. J Int Med Res 2001; 29: 13-21.
19. Borghi C, Boschi S, Ambrosioni E et al. Evidence of a partial escape of renin-angiotensin-aldosterone blockade in patients with acute myocardial infarction treated with ACE inhibitors. J Clin Pharmacol 1993; 33: 40-5.

20. Pitt B, Remme W, Zannad F, Neaton J, Martínez F, RONIKER B ET AL. Eplerenone, a selective aldosterone blocker, in patients with left ventricular dysfunction after myocardial infarction. N Engl J Med 2003; 348: 1309-21.

21. Pfefferer MA, McMurray J, Velázquez E, Rouleau JL, Køber L, Maggioni A et al. Valsartan, Captopril, or Both in Myocardial Infarction Complicated by Heart Failure, Left Ventricular Dysfunction, or Both. N Engl J Med 2003; 349: 1893-906.

22. PALMER BF. Managing Hyperkalemia Caused by Inhibitors of the Renin-Angiotensin-Aldosterone System. N Engl J Med 2004; 351: 585-92.

23. Juurink D, Mamdani MM, Lee DS, Kopp A, Austin PO, Laupacis A Et al. Rates of Hyperkalemia after Publication of the Randomized Aldactone Evaluation Study. N Engl J Med 2004; 351: 543-51.

24. REARDON LC, MACPHERSON DS. Hyperkalemia in outpatients using angiotensin-converting enzyme inhibitors. Arch Intern Med 1998; 158: 26-32.

25. Acker CG, Johnson JP, Palevsky PM, Greenberg A. Hyperkalemia in hospitalized patients: causes, adequacy of treatment, and results of an attempt to improve physician compliance with published therapy guidelines. Arch Intern Med 1998; 158: 917-24.

26. Wrenger E, MüLer R, Moesenthin M, Welte T, FRÖLCH J, NeUMANN K. Interaction of spironolactone with ACE inhibitors or angiotensin receptor blockers: analysis of 44 cases. BMJ 2003; 327: 147-9.

27. PRY Bys KM. Deadly drug interactions in emergency medicine. Emerg Med Clin N Am 2004; 22: 845-63. 